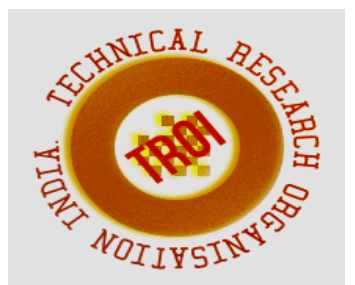

\title{
A MULTISCALE APPROACH BASED AUTOMATIC SHIP
} DETECTION

\author{
${ }^{\# 1}$ Associate Prof. Vijaya Geetha. R, ${ }^{\# 2}$ Chetan. S, ${ }^{\# 2}$ K. Deepika, ${ }^{\# 2}$ Kishore. R, ${ }^{\# 2}$ Likitha.S \\ ${ }^{1}$ Dept of Electronics and Communication Engineering, Dr. T.Thimmaiah Institute of Technology, \\ Visvesvaraya Technological University, Belagavi, Karnataka, India \\ vijayageethabe@gmail.com \\ ${ }^{2}$ Dept of Electronics and Communication Engineering, Dr. T.Thimmaiah Institute of Technology, \\ Visvesvaraya Technological University, Belagavi, Karnataka, India \\ kishorereddy27.kv@gmail.com
}

\section{Abstract}

This paper presents a new method for ship detection. It uses SAR image obtained by passing pulses of microwave signal and recording the reflected signal. SAR image is used for ship detection because it functions irrespective of weather conditions even at day and night. Speckle noise is inbuilt in SAR images are formed by recording the radar echo signals of EM signal multiplicative with granular noise;this noise degrades the quality of SAR images. Preprocessing is done using Lee filter to remove the speckle noise. Quality measurement like SNR, PSNR, SSIM and ENL is done. Image profile is verified to find effective noise suppression. After pre-processing the SAR image is enhanced using DWT (discrete wavelet transform) this wavelet transform preserves high frequency information and reduces artifacts. Enhanced image consists of many information from which the relevant image has to be extracted this process is called as feature extraction, feature extraction is done using k-means Clustering algorithm were the given data is grouped into $k$ number of clusters. Finally, the ship detection image is obtained and it is compared with the ground truth image, quantitatively the ship Detection image is evaluated using confusion matrix. The Outcomes obtained from confusion matrix are True Positive, True Negative, False Positive, False Negative, Sensitivity, Specificity, Precision, False positive rate, Error rate, Accuracy, False alarm rate.
Keyword: SAR image, Lee filter, discrete wavelet transform(DWT), k-means clustering algorithm and performance measures.

\section{I.INTRODUCTION}

Ship detection plays an important role in maritime piracy, Border control, defence and maritime security, maritime spatial Planning etc. Ship can be easily identified in image taken from Space, These two or three dimensional images are formed by sending Pulses of electromagnetic radiations in the microwave wavelength region from the radar which is on board, these pulses are received and recorded to form the high resolution Synthetic Aperture Radar (SAR) image are - RADARSAT-1, RADARSAT-2, TerraSAR- $X$, Tandem-X, COSMO SKYMed 1, 2, 3 and 4 sentinel-1A and sentinel-1B. SAR image are less influenced by the time and weather conditions than the optical images, SAR sensors carries effectiveness at any time of the day and night. Hence, SAR image promote the development of new automatic ship detection. We propose a system to detect the ships using synthetic aperture radar images. The SAR images are preprocessed using enhanced Lee filter to remove the speckle noise. The output of the lee filter is enhanced by DWT and further feature extraction is done using k-means clustering algorithm to extract the ship from the complex background. 


\section{II.LITERATURE SURVEY}

Input image is given to the system which has been trained using neural network to detect the ship from entire matrix of image, feature extraction is done using decision boundary feature extraction (DBFE) [1]. The most highlighted disadvantage is that the ship at port or near the dock cannot be correctlyclassified most of the times.A novel ship detection method makes use of constant false alarm rate(CFAR) pre-screening followed by a cascade classified ship discriminator. Ship are identified by haar-like feature using adaptive boosting training on the classified with an accuracy of $89.38 \%$ and FAR of $1.47^{\wedge}-8$ and MCC( matthew correlation coefficient) of 0.91[2].MLCC algorithm extracts the images of ships embedded in radar clutter by thresholding coherence image produced by cross-correlating SAR multi-look sub-images [3]. It is difficult to determine the correct threshold values in automatic manner.

Wavelet decomposition is used to detect ships of any size in both optical and SAR imagery[4]. Difficult to process very high resolution optical images with whitecaps, even if the ships are detected it has more false alarm due to whitecaps DT-CWT highlight superior performance in parameter such as PSNR, RMSE compared to DWT and DWTSWTMultiscale heterogeneities and contrario decision is used to detect ship[6]. The proposed method not only detects ships of different size but also has better performance than previous ship detection.

Variance heighted information entropy (VWIE) method is used to find dissimilarity between target and its neighbourhood [7] window selection mechanism based on multiscale local contrast method is used to enhance target; this approach can detect ships from complex background SAR images.

Ship detection process is divided into two stages detection and discrimination. In this detection stage the hermition product is extended to obtain a new detector. In the discrimination stage a covariance descriptor is used [8]. Result show that this scheme can ships with less false alarms.

In the first step feature extraction is done by analysing high- resolution SAR images, kernel density function (KDE), aspect ratio (AR), and ship pixels(SP)[9]. This method guarantees a high estimation of target detection probability and a low estimation of undetected target probability.

Ship detection is based on two steps: prescreening and discrimination. In the prescreening Stage classification between uniform and non-uniform part of the image is done by selecting the region of interest discrimination is done for better representation target in both shape and texture[10]. Main drawback is that separate machine learning code has to be written.

\section{METHODOLOGY}

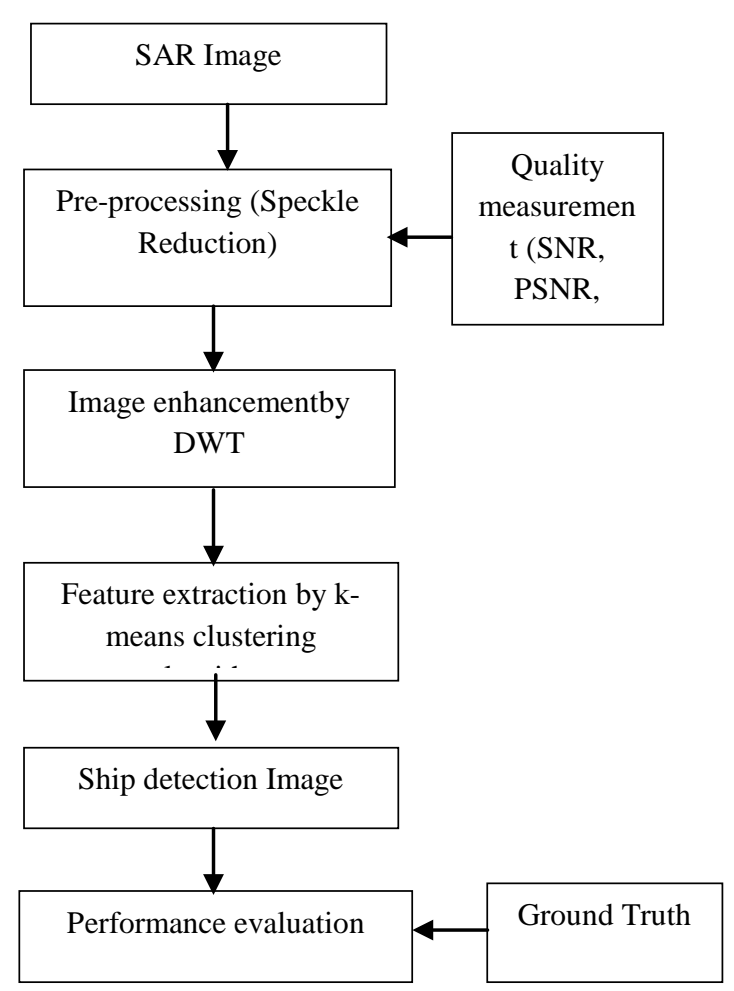

Fig.1. General over view of ship detection

SAR image is formed by transmitting receiving and recording successive pulse of electromagnetic waves, these pulses are transmitted from an air borne object. The position of air borne object change with respect to the ship target so the electromagnetic waves interfere with each light and dark pixels known as speckle noise.This noise target the quality of SAR images which leads to false target detection so, the obtained raw data has to be pre-processed.

\section{a. Pre-processing}

Speckle noise is a multiplicative noise which causes difficulties during the process of analysis of SAR images like reduces important information from image as edge, shape, 
intensity value etc. Hence this reduction has to be deployed prior to the use of SAR images.

Pre-processing is the step were the unwanted distortions (speckle noise) that suppresses the image data is removed using filter which is also called as despeckling. Speckle noise is removed by using lee filter.

Lee filter preserves the details in an image, it works on the basis of variance which means is the performs smoothing for region with low variance and not for high variance. Lee filter preserves the details in low as well as high contrast regions hence it is called as an adaptive filter. Lee filter model is defined by,

$I_{m g}(i, j)=I_{m}+W^{*}\left(C_{p}-I_{m}\right)(1)$

Where,

$I_{m g}=$ pixel value after filtering, $I_{m}=$ mean intensity of filter window, $C_{p}=$ centre pixel $W=$ Filter window, $W=\sigma^{2}\left(\sigma^{2}+\rho^{2}\right), \sigma^{2}$ is the variance of the pixel, $\sigma^{2}=\frac{1}{N} \sum_{j=0}^{N-1}\left(X_{j}\right)^{2}, \mathrm{~N}=$ Size of the filter, $X_{j}=$ pixel value at $\mathrm{j}, \rho=$ Additive noise variance ,

$$
\rho^{2}=\frac{1}{M} \sum_{j=0}^{M-1}\left(Y_{j}\right)^{2}
$$

Quality of the lee filter is measured using parameters such as SNR, PSNR, SSIM and ENL.

1. SNR (signal to noise ratio): It is the ratio of desired signal to level of noise. It has higher value for lower speckle.

$\mathrm{SNR}=10 \cdot \log _{10} \frac{\sum_{\mathrm{i}=1}^{\mathrm{M}} \sum_{\mathrm{j}=1}^{\mathrm{N}}\left(\mathrm{x}_{\mathrm{i}, \mathrm{j}}^{2}+\mathrm{y}_{\mathrm{i}, \mathrm{j}}^{2}\right)}{\sum_{\mathrm{i}=1}^{\mathrm{M}} \sum_{\mathrm{j}=1}^{\mathrm{N}}\left(\mathrm{x}_{\mathrm{i}, \mathrm{j}}+\mathrm{y}_{\mathrm{i}, \mathrm{j}}\right)^{2}}$

Where i, j represents rows and columns respectively.

2. PSNR(peak signal to noise ratio): It is the ratio of power of signal to the power of corrupting noise.

PSNR $=10 \log _{10} \frac{L^{2}}{\mathrm{MSE}}(3)$

Where, MSE is mean square error (MSE) which is defined as the difference between the mean squared value of original to despeckled image. $\mathrm{L}$ is image pixel intensity MSE is given by,

$\operatorname{MSE}=\frac{1}{\mathrm{MN}} \sum_{i=0}^{\mathrm{M}-1} \sum_{j=0}^{\mathrm{N}-1}[\mathrm{I}(\mathrm{i}, \mathrm{j})-\hat{\mathrm{I}}(\mathrm{i}, \mathrm{j})]^{2}(4)$
Where $\mathrm{M}$ and $\mathrm{N}$ are number of rows and columns $I(i, j)$ and $\hat{I}(i, j)$ are original and despeckled image respectively.

3. SSIM (Structural similarity index): It is the measure of structure similarity between the original and despeckled image. It is given by,

$\operatorname{SSIM}(x, y)=\frac{\left(2 \mu_{x} \mu_{y}+C_{1}\right)\left(2 \sigma_{x y}+C_{2}\right)}{\left(\mu_{x}^{2}+\mu_{y}^{2}+C_{1}\right)\left(\sigma_{x}^{2}+\sigma_{y}^{2}+C_{2}\right)}(5)$

Where $C_{1}=\left(K_{1} L\right)^{2} ; \quad C_{2}=\left(K_{2} L\right)^{2}$ these two variables stabilize the division with weak denominator. $\mathrm{L}$ is dynamic range. $\mu_{x}, \mu_{y}, \sigma_{x}^{2}$ and $\sigma_{y}^{2}$ are array of $\mathrm{x}$ and $\mathrm{y}$ and variance of $\mathrm{x}$ and y respectively.

After pre-processing image is enhanced using DWT.

\section{b. Image enhancement by DWT}

Despeckled image is enhanced to make it more suitable for further processing. Image enhancement consist of a collection of techniques that improve the appearance of an image. This paper uses discrete wavelet transform as the enhancement method whose key feature is image decomposition and reconstruction. Decomposition and reconstruction is done by filtering the image through low pass and high pass filter and then sampling.

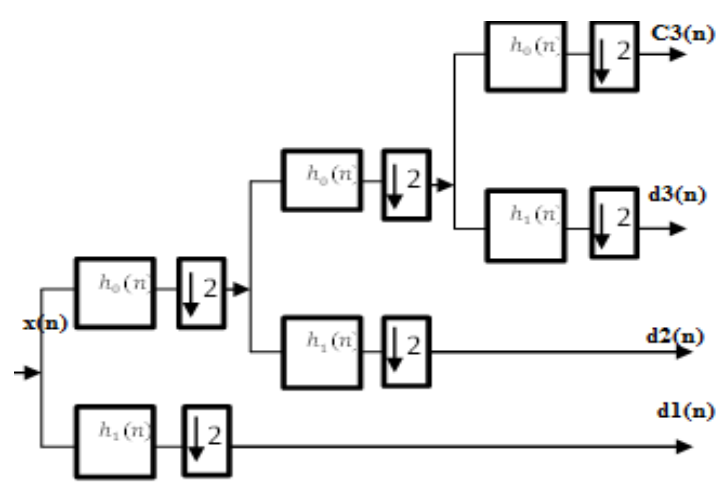

Fig.2 Illustration of DWT

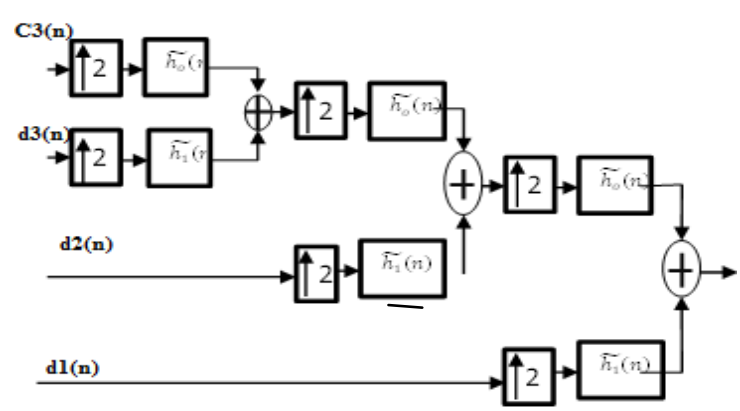

Fig.3 Illustration of IDWT 
From the figure image decomposition is done by passing the image through low pass and high pass filter $h_{0}(n)$ and $h_{1}(n)$ respectively and then down sampling by factor of 2 . The input image is decomposed into four sub bands; the four sub bands are recursively combined to reconstruct the original image.

$C_{3}(n), d_{1}(n), d_{2}(n)$ and $d_{3}(n)$ represents four subbands obtained by image decomposition.

$\check{x}(n)$ represents the reconstructed image, from the reconstructed image relevant data is extracted using $\mathrm{k}$ means clustering algorithm.

\section{c. Feature extraction by K-means clustering}

The process of getting the most relevant data from the enhanced image is called as feature extraction. K-means clustering is one of the easiest feature extraction technique. It consists of the following steps:

Step 1: Define the centroids for all the clusters

Step 2: Assign each group to the nearest centroid

Step 3: When all the groups are assigned, calculate the positions of the centroids

Step 4: Repeat step 2 and 3 until the centroids doesn't change its position

This produces the minimized number of clusters from which the relevant feature can be extracted.

The objective function for the k-means clustering algorithm is given by,

$j=\sum_{j=1}^{k} \sum_{i=1}^{n}\left\|x_{i}^{(j)}-c_{j}\right\|^{2}(6)$

Where,

$\left\|x_{i}^{(j)}-c_{j}\right\|^{2}$ Is a distance between a data point $x_{i}^{(j)}$ and the cluster centre, $c_{j}$ is the distance of the $\mathrm{n}$ data points from their respective cluster centre.

\section{d. Performance evaluation}

Confusion matrix is a quantitative method of calculating the image classification accuracy it has four outcomes- true positive, true negative, false positive, false negative. The various measures obtained from confusion matrix are: error rate, accuracy, precision,

True positive(TP): The number of samples correctly marked as positive i.e. the samples that are present in the detected ship image as well as the ground truth image is called as true positive or correct positive prediction.

False positive(FP): The number of samples incorrectly marked as positive. The samples are not present in the ground truth image but it is present in the detected ship image is called as false positive or incorrect positive prediction.

True negative(TN): The number of samples correctly marked as negative i.e. the sample that are not present in both ground truth image and detected ship image is called true negative or correct negative prediction.

False negative(FN): The number of samples correctly marked as negative is called as false positive or incorrect negative prediction.

\section{RESULTS AND DISCUSSION}

Speckle noise usually consists of high frequency components which have to be removed by using low pass filter. Lee filter shows better result when compared with other adaptive filters in terms of preserving details. SAR images were pre-processed individually by four adaptive filters (Kaun, Wiener, Frost and Lee) to remove noise. Lee filter shows better results than the other filters in edge preservation. Lee filter works on the variance basis, i.e. it performs smoothing if the variance of the area is low. Lee filter can preserve details in low as well as in high contrast hence it is adaptive in nature.

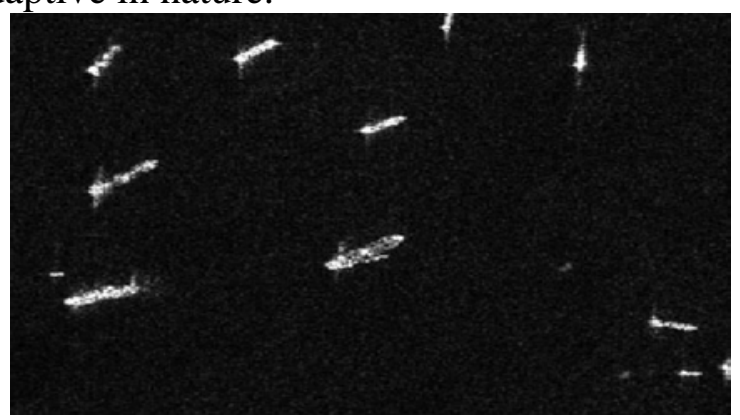

Fig.2. Input image

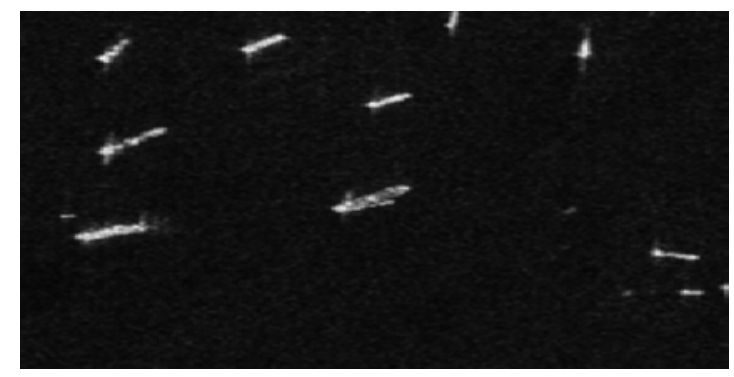

Fig.3. Despeckled image 
After pre-processing to measure the quality of despeckling the quality measurements like SNR, PSNR, SSIM, and ENL were calculated and the tabulation of the result obtained is as shown below.

Table.1. Quality measurements

\begin{tabular}{|c|c|c|c|c|}
\hline Quality & SNR & PSNR & SSIM & ENL \\
\hline Frost & 10.7122 & 25.89191 & 0.7542 & 36.8045 \\
\hline Kuan & 10.8304 & 26.15196 & 0.7729 & 28.4846 \\
\hline Lee & 11.2110 & 26.66983 & 0.7850 & 38.39 \\
\hline Wiener & 17.8204 & 28.6722 & 0.8107 & 42.74 \\
\hline
\end{tabular}

After quality measurements image profile was The quality measurements of Lee filter and obtained for all the four adaptive filters. Image Wiener filter show that it has better profile retrieves the intensity values of pixels performance in terms of SNR, PSNR, SSIM and along a line or multipath in grey scale, binary or ENL, but when image profile is taken into RGB image in the current axes and display a plot of the intensity values. If a single line segment is selected, then image profile creates two dimensional plot of intensity value versus distance along the line segment. If the path consists of two or more-line segment image profile creates a three dimensional plot of the intensity value versus their $\mathrm{X}$ and $\mathrm{Y}$ coordinates. Image profile for Lee filter and wiener filter is as shown in Fig. 4.
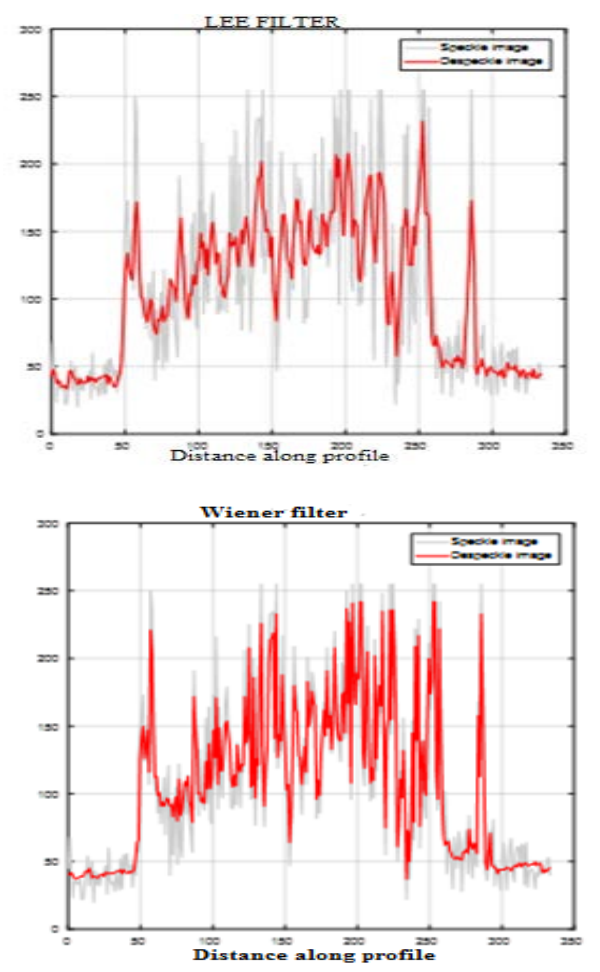

consideration Lee filter performs better. Speckle noise increases the intensity values is due to which there are many peaks in the image and these are represented as grey plot in the image profile shown in Fig. 4.3. The red colour plot in the image shows the despeckled image intensity value, this comparison between the Lee and wiener filter shows that Lee filter reduces the speckle noise better than wiener. Lee filter preserves the Low intensity regions were as wiener filter smoothens and causes feature broadening affect which increases the complexity of ship detection.Hence, Lee filter is used at the pre-processing stage to remove speckle noise. The pre-processed image is enhanced by DWT(Discrete wavelet transform) it uses multi-resolution filter banks and special wavelet filters for the analysis and reconstruction of signals. The output of DWT has four sub bands(LL, HH, LH and HL). The enhanced images are as following

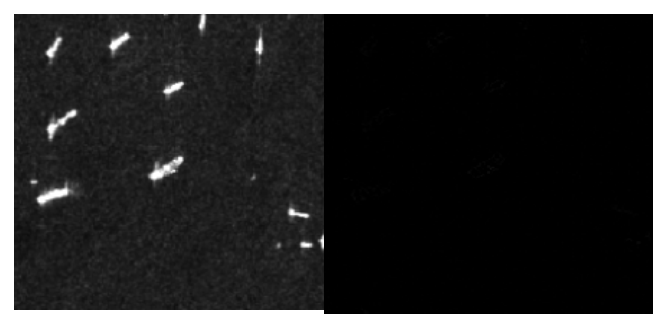

(a)

(b)

Fig.4. Comparison of image profile for Lee and Wiener filter 


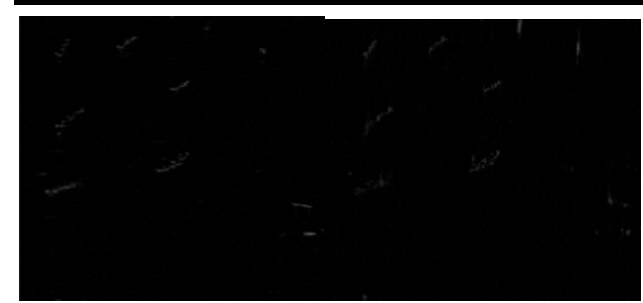

(c)

(d)

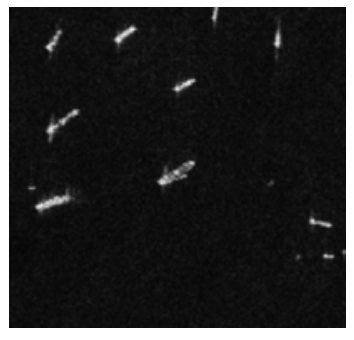

(e)

Fig.5. (a) LL band image (b) HH band image (c) LH band image (d) HL band image (e) reconstructed image

Inverse of DWT is performed to reconstruct the image.

The reconstructed image is split into clusters by applying k-means clustering algorithm. The obtained output is as shown below

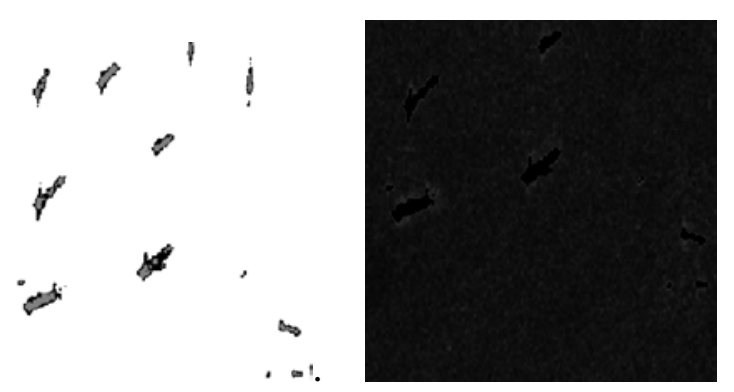

(a)

(b)

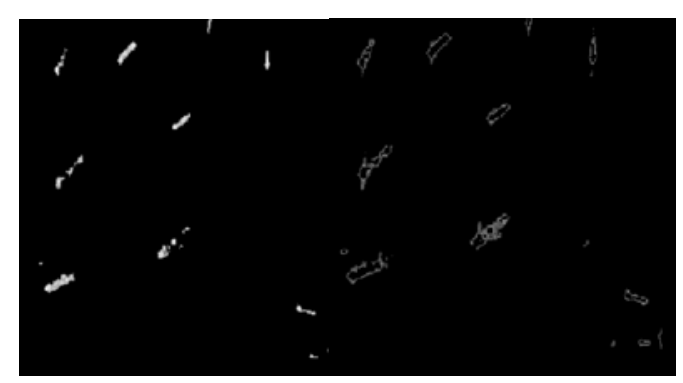

(c)

(d)

Fig.6. Output of $k$-means clustering (a) Image labelled by cluster index (b) Object incluster 1

(c) object in cluster 2 (d) object in cluster 3

The output of k-means clustering algorithm is the ship detection image, to check the accuracy of classification confusion matrix is used which has four outcomes- true positive, true negative, false positive and false negative. The various parameters that can be calculated using these outcomes are accuracy, false alarm rate, error rate and precision. The classificationaccuracy of the obtainedresults shows that the confusion matrix is the better classification method.

Table.2. Comparison of accuracy of classification of the proposed method with MSKI

\begin{tabular}{|c|c|c|}
\hline Methods & MSKI & $\begin{array}{l}\text { Proposed } \\
\text { Algorithm }\end{array}$ \\
\hline $\begin{array}{l}\text { False } \\
\text { Positive }\end{array}$ & 5918 & 1284 \\
\hline $\begin{array}{l}\text { False } \\
\text { Negative }\end{array}$ & 43 & 49 \\
\hline $\begin{array}{l}\text { True } \\
\text { Positive }\end{array}$ & 256 & 243 \\
\hline $\begin{array}{l}\text { True } \\
\text { Negative }\end{array}$ & 32199 & 36840 \\
\hline Accuracy & 0.8448 & 0.9653 \\
\hline $\begin{array}{l}\text { Error } \\
\text { Rate }\end{array}$ & 0.1552 & 0.0347 \\
\hline $\begin{array}{l}\text { False } \\
\text { Alarm Rate }\end{array}$ & 0.1553 & 0.0337 \\
\hline Precision & 0.0415 & 0.1591 \\
\hline
\end{tabular}

\section{REFERENCES}

[1.] Akshay Mutalikdesai et al., "Machine learningapproach for ship detection using remotely sensed images" $2^{\text {nd }}$ international conference for convergence in technology(I2CT) 2017.

[2.] C. P. Schwegmann et al., "Synthetic aperture radar ship detection using Haar-Like Features” IEEE geoscience and remote sensing letters 2016.

[3.] Kazuo Ouchi et al., "Improvement of ship detection accuracy by SAR multilook Cross- Correlation technique using adaptive CFAR” IEEE2010.

[4.] G. Jubelin et al., “ Multiscale algorithm for ship detection in mid, high and very high resolution optical imagery" IEEE 2014. 
[5.] M. Hemalatha et al., "Comparison of DWT, DWT-SWT, and DT-CWT for low resolution satellite images enhancement” IEEE transaction 2013.

[6.] Xiaolong Wang et al., "Ship detection for complex background SAR images based on a multiscale variance weighted image entropy method” IEEE geoscience and letters 2016.
[7.] Chao Wang et al., "Ship detection for high- resolution SAR images based on feature analysis" IEEE geoscience and remote sensing letters JAN 2014.

[8.] Chan Dong et al., "Ship detection in optical remote sensing images based on saliency and a rotation-invariant descriptor” remote sensing article 2018. 